\title{
Routine use of hemiarch during acute type A aortic dissection repair
}

\author{
Ibrahim Sultan*, Jeremy McGarvey*, Prashanth Vallabhajosyula, Nimesh D. Desai, Joseph E. Bavaria, \\ Wilson Y. Szeto \\ Division of Cardiovascular Surgery, University of Pennsylvania Medical Center, Philadelphia, PA, USA \\ *These authors contributed equally to this work. \\ Correspondence to: Wilson Y. Szeto. Division of Cardiovascular Surgery, University of Pennsylvania Medical Center, Penn Presbyterian Medical \\ Center, 51 N 39th St, Heart \& Vascular Pavilion 2A, Philadelphia, PA 19104, USA. Email: wilson.szeto@uphs.upenn.edu.
}

Submitted Jan 06, 2016. Accepted for publication Apr 08, 2016.

doi: $10.21037 /$ acs.2016.04.01

View this article at: http://dx.doi.org/10.21037/acs.2016.04.01

\section{Clinical vignette}

A 52-year-old man with a history of hypertension and $85 \%$ right-sided internal carotid stenosis presented to the emergency department with chest pain and lower extremity malperfusion with loss of right femoral pulse. He underwent a computed tomography (CT) scan that indicated an acute type A dissection (DeBakey I) and was directly transferred to our operating room.

\section{Surgical techniques}

\section{Preparation}

Bilateral radial arterial lines were placed. The patient underwent standard induction for general anesthesia and a large bore right internal jugular catheter with a pulmonary artery catheter was placed. Near infrared spectroscopy (NIRS) and electroencephalogram (EEG) are employed routinely for intraoperative monitoring in all arch cases at our institution. However, due to the emergent nature, only NIRS was available for this patient. Transesophageal echocardiography (TEE) was performed to confirm the diagnosis and to evaluate for any other valvular issues and cardiac function.

\section{Exposition}

We approach all patients with a standard median sternotomy. At our center, there are variations in cerebral protection strategy and circulation management based on patient presentation and surgeon preference, ranging from retrograde cerebral perfusion (RCP) and deep hypothermic circulatory arrest (DHCP) to antegrade cerebral perfusion (ACP) and moderate hypothermic circulatory arrest (MHCA). For this patient presenting with acute malperfusion, the technique of choice was central aortic arterial cannulation and MHCA with ACP during hemiarch reconstruction in an effort to minimize malperfusion insult and expedite surgical reconstruction of the arch.

\section{Operation}

The operation was begun with systemic heparinization after the pericardium was opened (Video 1). Based on the CT scan and TEE, we identified a cannulation site on the ascending aorta along the lesser curve to ensure cannulation of the true lumen. Using a Seldinger technique with an 18 gauge needle under TEE guidance, the aorta was punctured with confirmation of the wire placement and subsequently the arterial cannula in the true lumen. We prefer to use the OptiSite ${ }^{\mathrm{TM}}$ Arterial Perfusion Cannula (Edwards, Lifesciences, Irvine) but any over the wire percutaneous 18 or 20 French $(\mathrm{Fr})$ arterial cannula can be used. The surgeon should feel two 'pops' with the needle as he or she enters from the aorta through the false lumen into the true lumen. A soft ' $j$ ' wire is then passed over the needle, which is identified in the true lumen on TEE (1). Venous cannulation was established with a dual stage venous cannula via the right atrium. A coronary sinus catheter was placed and subsequently used for intermittent cardioplegia. Cardiopulmonary bypass (CPB) was instituted, and a left ventricular vent was placed via the right superior pulmonary 
vein. The patient was cooled to 28 degrees Celsius. The aortopulmonary window was dissected out and cross clamp was applied.

After aortic crossclamp, the aorta was transected and antegrade ostial cardioplegia was given. The tear did not extend into the sinus segment and the aortic valve appeared normal on TEE and upon visualization. Therefore, we decided to spare the aortic valve and root in this patient. Once the core body temperature reached 28 degrees Celsius, we turned our attention to the arch.

The plane between the innominate artery and the left carotid was dissected to prepare a clamp site. If there is no hematoma or dissection of the innominate artery seen on CT and visual inspection, our ACP strategy is direct cannulation of the innominate artery (2). A 4-0 pledgeted polypropylene $\mathrm{U}$-stitch is placed on the innominate artery and a $9 \mathrm{Fr}$ aortic root cardioplegia catheter is inserted into the artery. This is deaired and connected to the cardioplegia system from the CPB machine.

In anticipation for MHCA, steroids are given to all patients, and the cross clamp and arterial cannula are removed. During MHCA, the arch is then opened and inspected for any tears. We resect the ascending aorta and arch aggressively, with the proximal resection to the base of the innominate artery and the distal resection to the lesser curve opposite the left subclavian artery, leaving the greater curve for our hemiarch reconstruction. At this point, the ACP line is deaired and the innominate artery is clamped. ACP is then instituted at a rate of $10-12 \mathrm{~mL} / \mathrm{kg}$ with a measurement of $\geq 45-50 \mathrm{mmHg}$ in the right radial arterial pressure line while monitoring NIRS and EEG for asymmetry. At this point, a decision is made whether the patient needs a hemiarch or a more aggressive total arch replacement (TAR) or a frozen elephant trunk (FET) with antegrade thoracic endovascular aortic replacement (TEVAR). If there is a tear of the greater curve of the arch, circumferential head vessel dissection, distal arch aneurysm or descending aortic pseudo coarctation (secondary to true lumen compression), a TAR or FET with TEVAR is considered. If the above are absent, we tend to favor a hemiarch over a TAR and FET with TEVAR (3).

The goal of the hemiarch reconstruction is to obliterate the false lumen and re-expand the true lumen distally. Using our "neo-media" technique, a piece of felt is trimmed and placed between the adventitia and intima of the arch. Subsequently, using a running 4-0 polypropylene, the wall of neo-hemiarch is constructed (4). The choice of the Dacron graft for the hemiarch reconstruction is then sized with an interannular valve sizer. This graft is beveled to reconstruct the curve of the arch and is then sewn to the hemiarch anastomosis with a running 4-0 polypropylene stitch. We make the anastomosis an on-lay intussuscepting anastomosis with the graft placed inside the aorta for optimal hemostasis. Once the anastomosis is complete, the graft is re-cannulated and deaired and CPB is reinstituted. The cardioplegia cannula is removed from the innominate artery and the $\mathrm{U}$ stitch is tied down. Hemostasis is confirmed by inspecting the entire suture line and repairing with any patch stitches if needed. The patient is rewarmed while proximal aortic work is completed.

\section{Completion}

When a valve resuspension is performed and the valve and the sinus segment are preserved, we typically use a single straight graft from the proximal aortic suture line to the hemiarch. However, in the setting of an aortic root replacement, separate grafts are required and a graft-to-graft anastomosis is performed. We rarely use any glue applicators or external felt with our anastomoses. We feel that the neomedia provides structure and strength to the dissected aorta, thereby improving hemostasis in our suture line.

\section{Comments}

\section{Clinical results}

Approximately $85 \%$ of patients who present with an acute type A dissection have had a hemiarch performed at our institution. This technique can be performed with low mortality and low arch reoperation rates. Performing a hemiarch does not eliminate the risk of distal aortic events but certainly decreases them. Freedom from distal aortic reintervention was $90 \%$ for DeBakey II dissection and $85 \%$ for DeBakey I dissection at 10 years (5).

\section{Advantages}

Innominate artery cannulation and MHCA decrease CPB and operative times and more importantly transfusion requirements. This has already been shown to be true in patients undergoing elective hemiarch reconstruction (2). The shorter time under circulatory arrest, the simplicity of performing a hemiarch, and the successful long-term outcomes of the technique with distal aortic remodeling all argue for performing a hemiarch. However, if we feel 
that a patient does need a TAR for the reasons mentioned previously, we do so without hesitation.

\section{Caveats}

It is important when cannulating the innominate artery to determine whether it has been dissected or not. This can be confirmed by CT scan and TEE in addition to visual inspection. If we feel that the innominate artery is dissected, we use balloon expanding cardioplegia lines that are inserted directly into the innominate artery and concomitantly in the left carotid artery (if needed). The presence of a distal arch aneurysm, true lumen compression causing distal aortic pseudocoarctation or a tear in the greater curvature of the arch prompt us to strongly consider a TAR or a FET with TEVAR instead of a hemiarch.

\section{Acknowledgements}

None.

\section{Footnote}

Conflicts of Interest: The authors have no conflicts of interest to declare.

\section{References}

1. Frederick JR, Yang E, Trubelja A, et al. Ascending aortic cannulation in acute type a dissection repair. Ann Thorac Surg 2013;95:1808-11.

2. Jassar AS, Vallabhajosyula P, Bavaria JE, et al. Direct innominate artery cannulation: An alternate technique for antegrade cerebral perfusion during aortic hemiarch reconstruction. J Thorac Cardiovasc Surg 2016;151:1073-8.

3. Sultan I, Szeto WY. Decision making in acute DeBakey I aortic dissection: Balancing extensive arch reconstruction versus mortality. J Thorac Cardiovasc Surg 2016;151:349-50.

4. Bavaria JE, Pochettino A, Brinster DR, et al. New paradigms and improved results for the surgical treatment of acute type A dissection. Ann Surg 2001;234:336-42; discussion 342-3.

5. Rylski B, Milewski RK, Bavaria JE, et al. Long-term results of aggressive hemiarch replacement in 534 patients with type A aortic dissection. J Thorac Cardiovasc Surg 2014;148:2981-5.
Cite this article as: Sultan I, McGarvey J, Vallabhajosyula P, Desai ND, Bavaria JE, Szeto WY. Routine use of hemiarch during acute type A aortic dissection repair. Ann Cardiothorac Surg 2016;5(3):245-247. doi: 10.21037/acs.2016.04.01 